\title{
Parosteal Lipoma in the Scapula Associated with a Osteochondroma- A Rare Case
}

\section{Fillho $\mathrm{DAO}^{1}$ and Lima $\mathrm{CMAO}^{2 *}$}

${ }^{1}$ Medical Radiologist, Alfredo Abraão Cancer Hospital, Brazil

${ }^{2}$ Medical Radiologist of UnitedHealth Group Inc. (UHG), Rede D'Or São Luiz and Home

Hospital Group, Radiology Assistant Professor of the Estácio de Sá Medical School, Student

Masters Course at the State University of Rio de Janeiro, Brazil

*Corresponding author: Cláudio Márcio Amaral de Oliveira Lima, MD, Medical Radiologist, House of Portugal Hospital, 72 Bishop's St, Rio de Janeiro, Brazil, 20261-064; Email: cmaolima@gmail.com

\section{Abstract}

Lipomas correspond to the most common tumors of the soft parts. Parosteal lipoma can manifest at any age, with a peak incidence between of 40 and 60 years old. It is a rare, benign, slow-growing tumor that grows exophytically from the periosteum. The most common sites are those that grow on the diaphyseal surface of long bones and extremities, such as the femur and proximal radius. The scapula is a very rare site of parosteal lipoma. In this study, we report a case of a parosteal lipoma growing up from the scapula associated with an osteochondroma in a 54-year-old female patient.

Keywords: Lipoma; Parosteal; Osteochondroma; Bone Tumor

Abbreviations: PL: Parosteal Lipomas; CT: Computed Tomography; MRI: Magnetic Resonance Imaging; LS: Liposarcoma; OC: Osteochondroma.

\section{Introduction}

Lipomas (LI) are benign tumors of mature adipose tissue with no cellular atypia, and they correspond to the most common tumors of the soft parts. Parosteal lipomas (PL) are rare, accounting for $0.3 \%$ of all LI. In about $50 \%$ of cases, the patients are aged between of 40 and 60 years old [1]. PL is a rare benign tumor that grows slow and exophytically from the periosteum and can have intervening thin septations [1]. The most frequently affected sites are the diaphyseal and metaphyseal regions of the long bones and this type of tumor tends to be indolent due to deep localization. The imaging features of PL are usually distinctive and are applicated simple radiography, computed tomography (CT) and magnetic resonance imaging (MRI). MRI is the most specific method to differentiate it from other tumors, such as welldifferentiated liposarcoma (LS) [1,2]. The scapula is a very rare site of $\mathrm{PL}$, and not many cases have been reported in the literature. In this study, we report a case of a PL growing from the scapula associated with a one osteochondroma $(\mathrm{OC})$ in a 54-year-old female patient.

\section{Case Report}

Female, 54 years of age, with a history of soft and painless tumor in the right dorsal region with an evolution of 4 years. The tumor had grown slowly over the years and had become a little more hardened. Negative fever or neurological deficit on physical examination, the patient had a palpable mass in the region adjacent to the lower border of the right scapula, with a predominantly softened consistency with no local inflammatory signs (Figure 1A). The x-ray showed an irregular bone protuberance in apparent contiguity with the inferior angle of the right scapula, bordered by a radiolucent 
image of regular and well defined contours (Figure 1B).

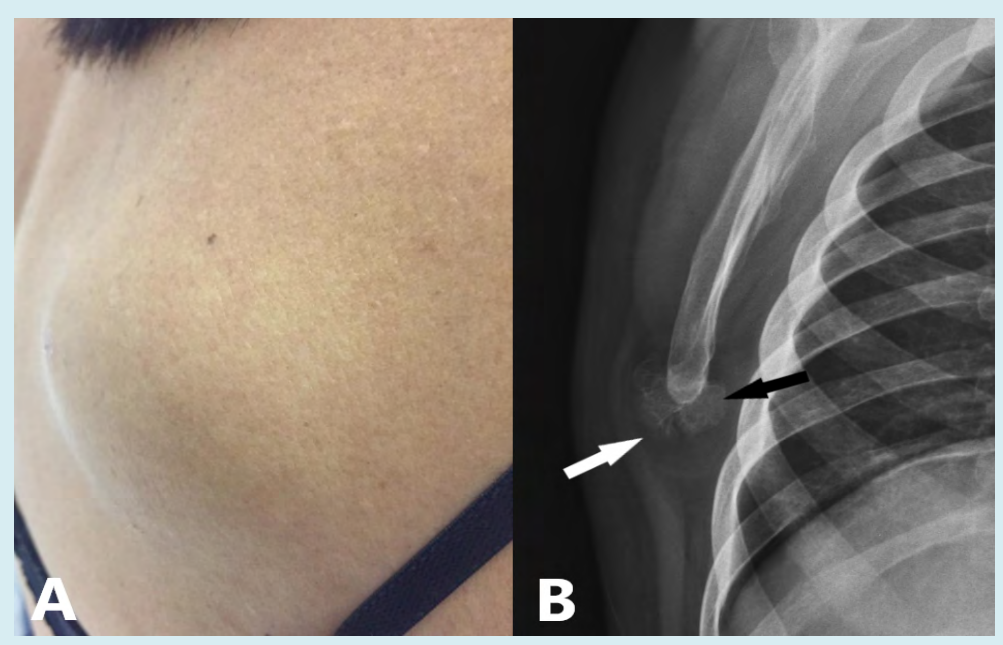

Figures 1A \& B: A) Tumoration of predominantly softened consistency, measuring approximately 6,0 x 5,0 cm, painless and without local inflammatory signs. B) X-ray of the right shoulder. Radiolucent lesion (white arrow) surrounding an irregular bone protuberance near the lower angle of the right scapula (black arrow).

CT with the intravenous contrast medium showed a bone excretion in the inferior angle of the right scapula in continuity with the bone marrow, which presents well defined contours, suggesting OC. Surrounding the lesion, there are an expansive formation with fat attenuation and no enhancement by the contrast medium (Figure 2). To better characterize the lesion, a MRI with gadolinium administration was performed, revealing an expansive formation that insinuated under the major rhomboid muscle, with hypersignal in the $\mathrm{T} 1$ and $\mathrm{T} 2$ weighted sequences, and suppression in the fat saturation sequences, characterizing fatty tissue. Associated to bone outgrowth in the inferior angle of the scapula, with lobulated margin and well defined, there are areas of hypersignal of permeio compatible with calcifications of probable nature chondral and a thin border of hyposignal, which may correspond to the cartilage layer, suggesting OC. A discrete enhancement by the paramagnetic contrast medium at the interface between the bone portion and the fatty component was observed suggesting fibrous tissue border (Figures $3 \& 4$ ).

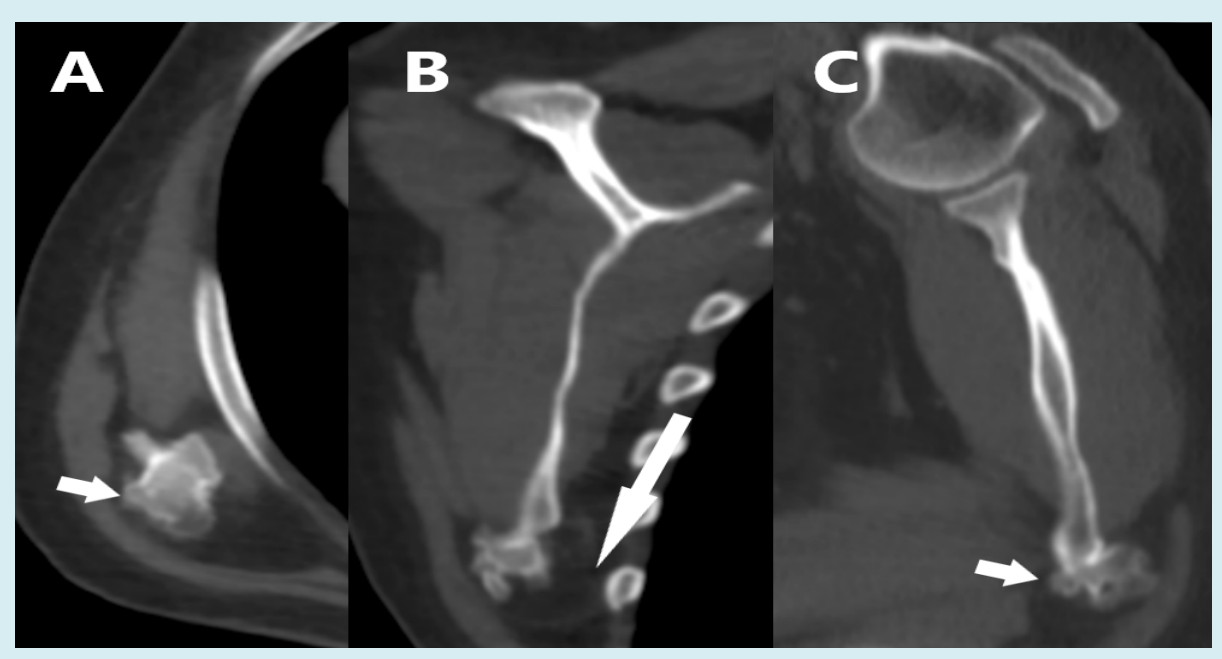

Figure 2: CT of the chest with a bone window A) axial plane, B) coronal plane and C) sagittal plane, Expansive formation with fat attenuation (long white arrow) associated with a bone excrescence in the inferior angle of the right scapula. The bone excretion presents continuity with the bone marrow of the scapula (short white arrows). 


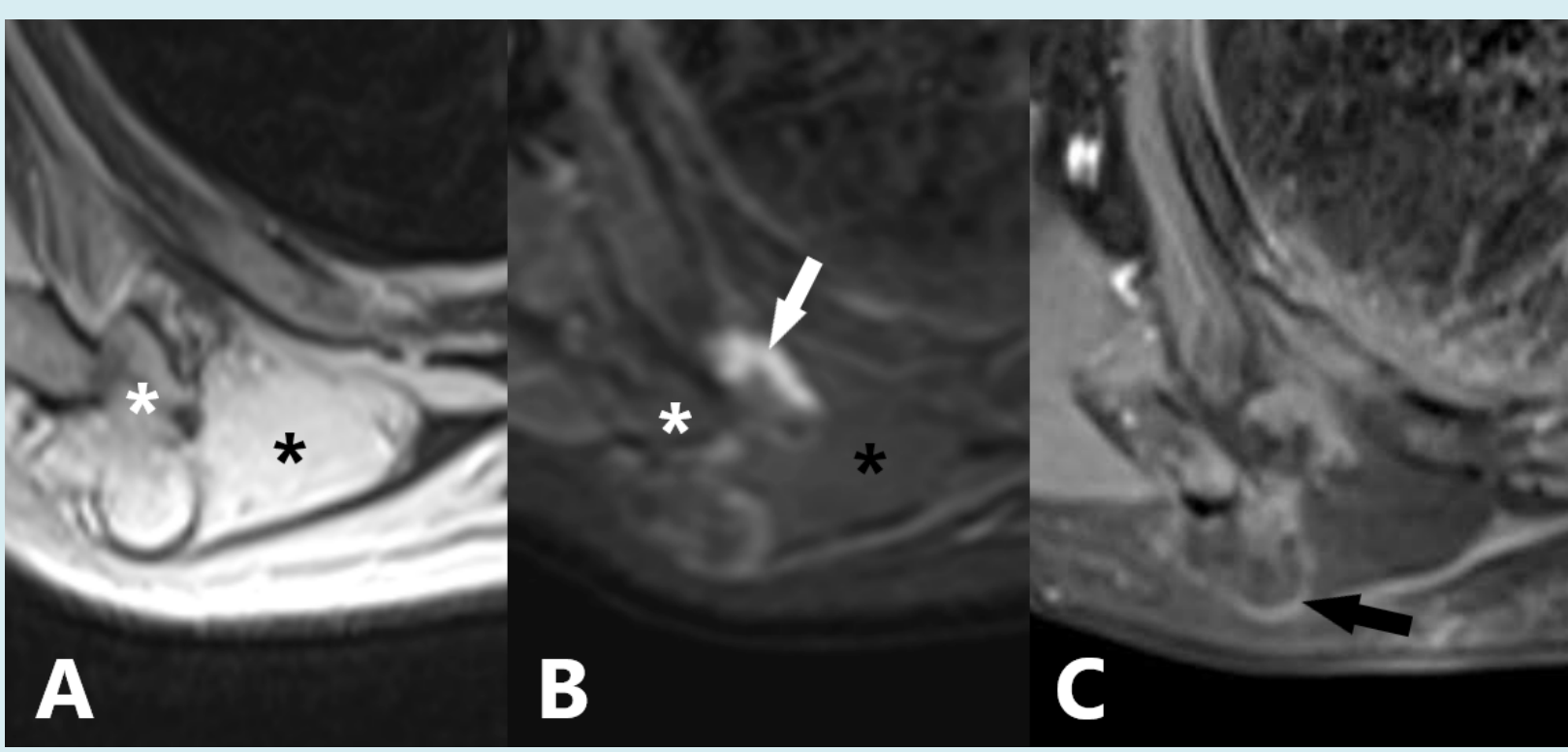

Figure 3: MRI: A) Axial T2 weighted, Expansive formation with high signal intensity (black asterisk) similar to subcutaneous fat and bone scoring in the inferior angle of the right scapula (white asterisk). The bone lesion presents continuity with the bone marrow of the scapula and a thin border of the low signal intensity being able to corresponds the cartilage layer, indicating a probable osteochondroma, B) Axial STIR. The fatty lesion presents suppression of the signal, characterizing lipomatous tissue (black asterist). Note that there is a linear hypersignal area at the anterior border of the bone lesion (white arrow), which corresponds to a probable hemorrhagic component secondary to the previous percutaneous biopsy procedure, C) Axial T1 weighted after administration of gadolinium. Note the fine area of the interface between the fatty and bony component, indicating that it is border of fibrous tissue (black arrow).

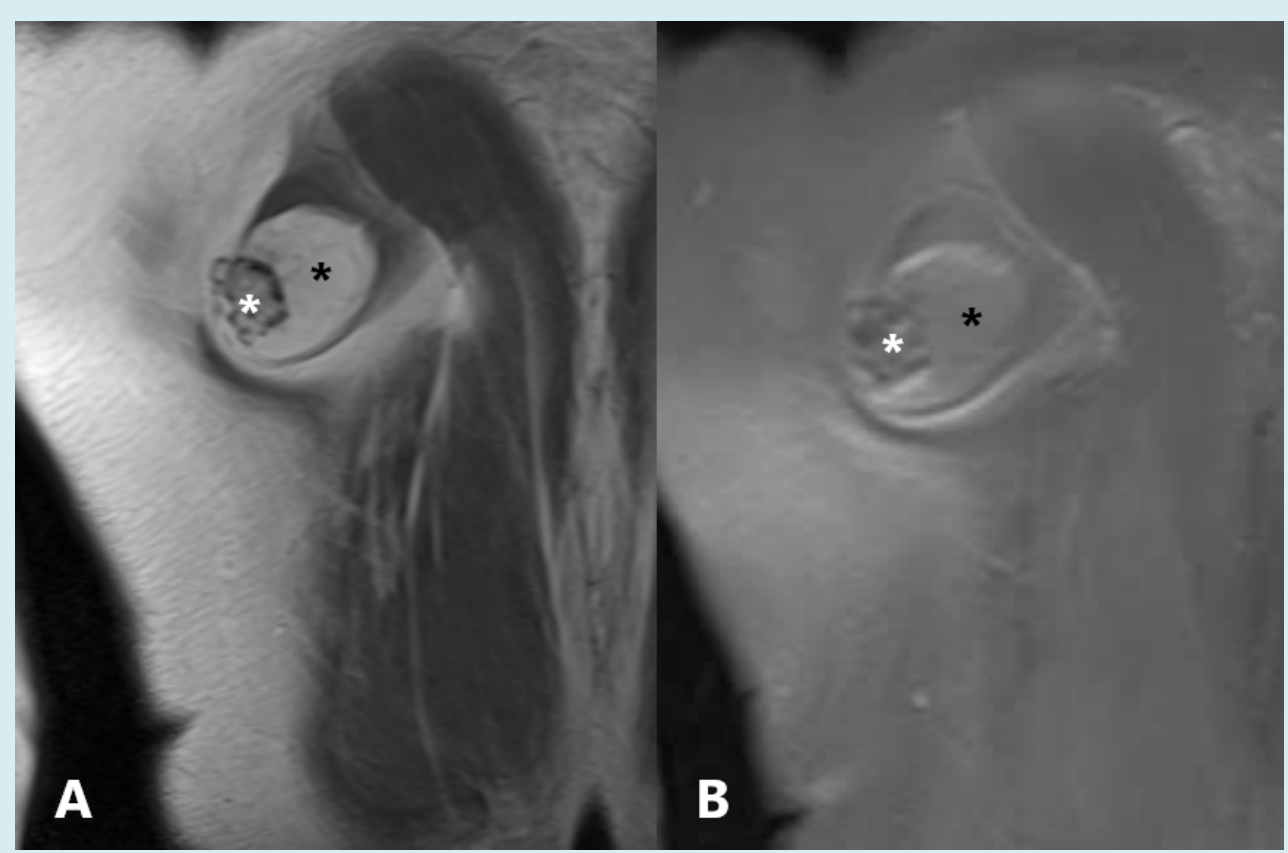

Figure 4: MRI: A) Coronal T1 weighted. Expansive formation with a hypersignal (black asterisk) similar to subcutaneous fat and a bone excrescence in the inferior angle of the right scapula (white asterisk), with small foci of hyposignal that can correspond to calcifications, B) Coronal T1 weighted fat-suppressed. The lesion presents suppression of the signal, characterizing fat (black asterisk). 


\section{Clinical Radiology \& Imaging Journal}

There was no evidence of neurovascular compression. The patient was submitted to a core-biopsy, which demonstrated adipose tissue without atypia and with some fibrotic beams (Figure 5), confirming the diagnosis of LI.
Therefore, the diagnosis of PL associated with an OC of the scapula was defined by the characteristic of the image and its location.

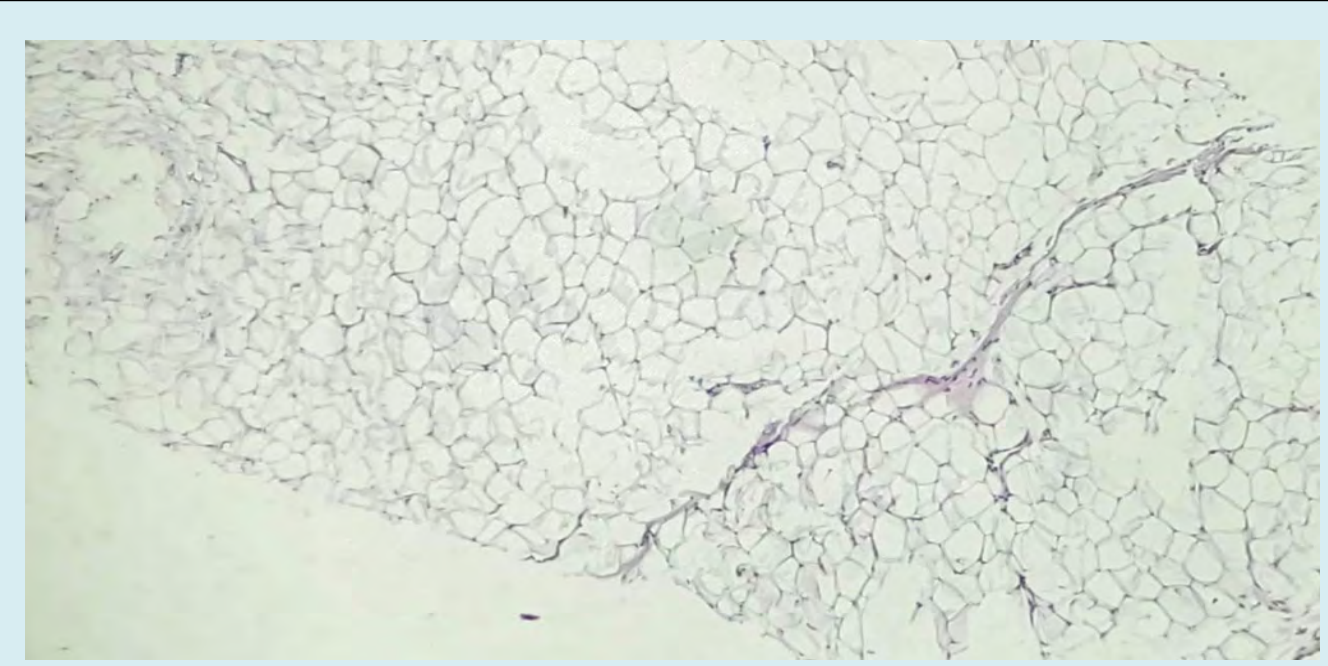

Figure 5: Histological study obtained by percutaneous core-biopsy, Cells of adipose tissue without atypia, with some fibrotic beams.

\section{Discussion}

LI are benign tumors of mature adipose tissue with no evidence of cellular atypia. They are the most common soft tissue tumors, accounting for up to $50 \%$ of all cases. PL is rare, accounting for $0.3 \%$ of all LI. It's can manifest at any age, with a peak incidence between 40 and 60 years old and affects on the same proportion men and women $[1,2]$. The first description of PL was published in German medical literature by Seerig in 1836 [6] and English medical literature in 1886 by Smith. However, the term PL was introduced by Power in 1888. PL is originating from mesenchymal cells of the periosteum. The periosteum does not have fat cells, so it is named as "PL" and not "periosteal LI". The relationship to the underlying bone distinguishes this lesion from a softtissue LI $[1,3,4]$. The most common sites are the femur, followed by the proximal radius. More rarely, these lesions have also been described on the clavicle, rib, scapula, pelvis, metacarpus, metatarsus, mandible and skull [2]. They present histopathological characteristics similar to all LI. PL may vary according to the degree of chondral modulation and endochondral ossification $[5,6]$.

In the simple radiography there are a well-defined radioluscentlipomatous mass with varying degrees of septation associated with surface bone productive changes ranging from very subtle to obvious cortical thickening and variable sizes of bone protuberances or excrescences $[2,7,8]$. May have adjacent bone changes most commonly hyperostosis reactions, cortical thickening, irregularity, bone excrescences, curvatures, cortical erosions or OC, as in our case [4]. At CT present fat density with a bone component adherent to the surface of the adjacent bone and is able to characterize the degree of septation and to define the relation of the mass with the underlying cortex, important for the surgical planning [2]. MRI is the most specific method to differentiate a PL from other tumors. It is observed a juxtacortical mass with hypersignal in both $\mathrm{T} 1$ and $\mathrm{T} 2$ weighted sequences, with suppression in the fat saturation sequences.

Occasionally, we can identify hyposignal streaks which correspond to fine fibrovascular tracts, which should not be confused with well differentiated LS, since they are thin and do not show enhancement by paramagnetic contrast medium $[5,8,9]$. The bone component presents low signal in T1 and T2. MRI is also able to identify muscle atrophy and signs of compression of adjacent neurovascular bundles [6,10-12]. PL can cause peripheral nerve compression, and there are reports in the literature of involvement of the radial, sciatic, ulnar and median nerve. Interestingly, the anchoring effect of the bone fixation site may predispose these pars plana LI to the mass effect and to the nerve impact, unlike the soft tissue LI pattern, which expand along the path of least resistance [2]. Post-contrast images may reveal enhancement at the interface of mass and bone protuberance, inferring in the possibility of fibrous tissue border or other soft tissue reactional changes $[7,10,11]$. 


\section{Clinical Radiology \& Imaging Journal}

In our case, near the lipomatous lesion, in the inferior angle of the right scapula, there was also a bone excrecence with characteristics compatible with OC. The treatment of choice for this mass is complete excision, which presents a good prognosis, with no recurrences described in the literature. PL does not present malignant potential, so conservative follow-up may be an option, in cases in which there is no neurovascular involvement [7]. Our patient refused the surgical treatment and is in a conservative follow-up.

\section{Conclusion}

PL is a rare benign tumor that grows from the periosteal layer and mainly affects long bones, such as the femur and proximal radius, but may also grow in more rare locations, such as the scapula, and may be associated with an OC or other adjacent bone changes. Imaging studies play an important role in the diagnosis and follow-up of the lesion. MRI is the most specific method to differentiate it from other tumors, such as well-differentiated LS. It also plays a fundamental role in the characterization of possible neurovascular compression and consequent muscular atrophy. The treatment of choice is resection; however, because it is a tumor with no malignant potential, image control may be an option in cases when there is no neurovascular compression.

\section{References}

1. Murphey MD, Carroll JF, Flemming DJ, Pope TL, Gannon FH et al. (2004) From the archives of the AFIP: benign musculoskeletal lipomatous lesions. Radiographics 24(5): 1433-1466.

2. Greco M, Mazzocchi M, Ribuffo D, Dessy LA, Scuderi N (2013) Parosteal lipoma: report of 15 new cases and a review of the literature. Ann Ital Chir 84(2): 229-235.

3. Myint ZW, Chow RD, Wang L, Chou PM (2015) Ossifying parosteal lipoma of the thoracic spine: a case report and review of literature. J Community Hosp Intern Med
Perspect 5(1): 26013.

4. Başarir K, Şahin E, Kalem M, Karaca MO, Yildiz Y, et al. (2017) Parosteal lipoma as a rare cause of peripheral neuropathy and local irritation: A report of 12 cases. Acta Orthop Traumatol Turc 51(6): 474-477.

5. Miller MD, Ragsdale BD, Sweet DE (1992) Parosteal lipomas: a new perspective. Pathology 24(3): 132-139.

6. Fleming RJ, Alpert M, Garcia A (1962) Parosteal lipoma. AJR Am J Roentgenol 87: 1075-1083.

7. Gholamrezanezhad A, Basques K, Kosmas C (2018) Peering beneath the surface: juxtacortical tumors of bone. Clin Imaging 50: 113-122.

8. Tetsuhiko G, Nakajima N, Yokota N, Yokomise H (2018) Thoracoscopic resection of parosteal lipoma of the rib using orthopedic electric micro drill. Gen Thorac Cardiovasc Surg 66(11): 675-678.

9. Ohguri T, Aoki T, Hisaoka M, Watanabe H, Nakamura K, et al. (2003) Differential diagnosis of benign peripheral lipoma from well-differentiated liposarcoma on MR imaging: is comparison of margins and internal characteristics useful?. AJR Am J Roentgenol 180(6): 1689-1694.

10. Balani A, Sankhe A, Dedhia T, Bhuta M, Lakhotia N, et al. (2014) Lump on Back: A Rare Case of Parosteal Lipoma of Scapula. Case Rep Radiol.

11. Yoon MA, Chung HW, Chee CG, Lee MH, Lee SH, et al. (2019) Risk Factors for Diagnostic Failure of UltrasoundGuided Core Needle Biopsy of Soft-Tissue Tumors Based on World Health Organization Classification Category and Biologic Potential. AJR Am J Roentgenol pp: 1-9.

12. Murugharaj S, Ahmed S, Abhay H, Najimudeen S (2019) Parosteal Lipoma of Proximal Radius: A Case Report of an Unusual Swelling and Review of Literature. J Orthop Case Rep 9(3): 46-48. 